\title{
Validación retrospectiva de la modificación del método bioquímico para la determinación de creatinina en suero, en un laboratorio clínico
}

\section{Retrospective validation of the modification of the biochemical method for the determination of serum creatinine, in a clinical laboratory}

\author{
Jesús E. Rodríguez-Villacis ${ }^{\mathrm{I}}$ \\ Universidad de Especialidades Espíritu Santo \\ jesusrodriguez@uees.edu.ec
}

Jorge Castillo-González II

Eduardo de la Torre-Quiñonez III

eduardofrancisco.delatorre@gmail.com

Manuel I. Guzmán-Veas ${ }^{\text {IV }}$

manuelguzmanveas@hotmail.com

Raisa S. Mariscal-García V

raisa.mariscal@hotmail.com

Walter E. Mariscal-Santi VI

Universidad de Guayaquil

walter.mariscals@ug.edu.ec

Frella S. García-Larreta VII

Universidad de Guayaquil

soraya.garcial@ug.edu.ec 


\section{Resumen}

El presente trabajo de titulación se basa en la sistematización de una experiencia que se originó en un periodo de pasantías realizada durante el mes de marzo de 2014 en un laboratorio clínico ubicado en el cantón General Villamil playas, centrándose en una validación retrospectiva, la importancia que tiene validar un método, y las mejoras que se dan al momento de entregar los resultados de los análisis que benefician tanto al laboratorio, como a sus pacientes. Encontraremos en este proyecto la problemática que tienen los laboratorios clínicos para adquirir una acreditación, antecedentes tanto internacionales como son la norma ISO 17025, norma ISO 151849, nacionales como la norma OAE GO4 ROO, dentro del laboratorio las buenas prácticas de laboratorio (BPL), su sistema de gestión de la calidad. Y la información obtenida durante este periodo de la experiencia. Todos desempeñando un papel importante para así lograr aportar al laboratorio la información necesaria para que alcance su objetivo final, la acreditación.

Palabras clave: Validación; modificación; acreditación; método bioquímico; laboratorio clínico; cantón playas; creatinina. 


\begin{abstract}
This work is based on degree systematization experience which originated in a period of internship made during march 2014 in a clinical laboratory located in canton General Villamil beaches, focusing on a retrospective validation the importance validate have a method and improvements to give dan when the test results that benefit both the laboratory as a patient. Find in this project having problems clinical laboratory accreditation to acquire both international background as are the ISO 17025, ISO standard 151849, standard national and ROO OAE GO4 within the laboratory the good laboratory practice (GLP) your system quality management. And information obtained during this period of experience. All performing an important role to achieve that contribute to the laboratory information necessary to reach your ultimate goal, the credit.
\end{abstract}

Key words: Validation; modification; accreditation; biochemical method; clinical laboratory; guangzhou beaches; creatinine. 


\section{Introducción.}

Es conveniente realizar esta sistematización de la validación retrospectiva de la variación en el método bioquímico para mostrar que efectivamente los resultados son seguros, precisos y confiables tanto para el Laboratorio Clínico, El Paciente y el Medico. (Perazzi \& Angerosa, 2011) (Myers, Miller, Coresh, Fleming, \& Greenberg, 2006)

La variación del método para determinación de creatinina ahorra tiempo, reactivos, recursos y talento humano, teniendo en cuenta que los resultados obtenidos sean eficaces. (Pennacchiotti, Benozzi, \& Ruiz, 2012)

$\mathrm{Al}$ acortar el método se logra que los resultados se obtengan más rápido para que el paciente los pueda retirar y de esta manera se mejora el servicio.

Una de las intenciones de este proyecto es entablar líneas de dialogo con el fabricante de los kits para determinación de creatinina, puesto que los análisis realizados durante varios años demuestran la eficacia y la eficiencia de la variación del método, pues se han realizado pruebas clínicas con el mismo suero control con las indicaciones del kit completo y en la variada obteniéndose los mismos resultados. (López, Blanes, \& Ríos, 2006)

La importancia de sistematizar la variación del método radica en el aporte en que este proceso tributa a la futura validación, misma que no sería posible si no se lograra establecer verificaciones continúas y precisas que garantizan que la información es fidedigna. (Flores, Alvo, \& Borja, 2009) 
El objetivo de la presente investigación es sistematizar la variación del método utilizado en el Laboratorio Clínico demostrando que las modificaciones no afectan la confiabilidad de los resultados.

\section{Metodología.}

\section{Enfoque metodológico}

El presente trabajo de investigación ha trabajado con el Análisis y Síntesis, como el método de guía en el estudio, por la descomposición de todos los factores que intervienen al momento de encajar en los indicadores a cumplir para la acreditación.

El tipo de investigación aplicado es Bibliográfica - Descriptiva por el importante aporte de información encontrada. En las Normas Ecuatorianas, Internacionales, On-line, Paginas Gubernamentales, y de la importante base de datos. Del Laboratorio Clínico ubicado en el Cantón General Villamil Playas.

\section{Enfoque de la investigación}

Esta investigación es de carácter Deconstructivo, seleccionado para llevar a cabo todo el proceso de sistematización que ha permitido arribar a una serie de conclusiones desde el momento en que se comenzó a recolectar la información, hasta cuando se obtuvieron los resultados y gráficos. Que nos permitieron concluir con el trabajo realizado y determinar que la técnica modifica comparada con la de la casa comercial, no variaron mucho en cuanto resultados.

\section{Población y muestra}


- Laboratorio Clínico ubicado en el Cantón General Villamil Playas.

- Base de datos del Laboratorio Clínico

- Al tratarse de un numero bajo de Población, la población es finita por lo tanto esta se convierte automáticamente en la Muestra.

\begin{tabular}{|c|c|}
\hline POBLACION & CANTIDAD \\
\hline JEFES DE LABORATORIOS & 15 \\
CLÍNICOS DEL CANTÓN & \\
GENERAL VILLAMIL & \\
PLAYAS & \\
\hline ANALISTAS DE & 28 \\
LABORATORIOS & \\
CLÍNICOS DEL CANTÓN & \\
GENERAL VILLAMIL & \\
PLAYAS & \\
\hline TOTAL & $\mathbf{4 3}$ \\
\hline
\end{tabular}

Técnicas y procedimientos

Técnicas de recolección de información

Se aplicó la técnica de la entrevista a un especialista en el área de acreditación de laboratorios clínicos.

Se realizaron encuestas a los Laboratorios Clínicos del Cantón General Villamil Playas para conocer su experiencia en la validación de métodos.

Se tomaron datos estadísticos. 


\section{Herramientas estadísticas}

Se elaboraron diseños de tablas estadísticas con la información obtenida, porcentajes, pasteles, curvas.

\section{Resultados.}

1. ¿Conoce usted lo que es una validación retrospectiva?

$\begin{array}{ccc}\text { PREGUNTA } & \text { SI } & \text { NO } \\ \text { JEFES DE } & 12 & 3 \\ \text { LABORATORIOS } & & \\ \begin{array}{c}\text { ANALISTAS DE } \\ \text { LABORATORIOS } \\ \text { TOTAL } \%\end{array} & 15 & 13 \\ & 62.79 \% & 37.21 \%\end{array}$

Del total de la Población encuestada el $62.79 \%$ conocía del tema mientras que el $37.21 \%$ No.

2.- ¿Cree usted que los laboratorios clínicos deben llevar banco de datos que sirvan como referencia estadística para una acreditación?

$\begin{array}{ccc}\text { PREGUNTA } & \text { SI } & \text { NO } \\ \text { JEFES DE } & 15 & 0 \\ \text { LABORATORIOS } & & \\ \begin{array}{c}\text { ANALISTAS DE } \\ \text { LABORATORIOS } \\ \text { TOTAL } \%\end{array} & 28 & 0 \\ & 100 \% & 0 \%\end{array}$

Del total de la Población encuestada el 100\% conocía del tema mientras que el 0\% No. 
3.- ¿Confía usted en los resultados que se obtienen mediante los kits de Reactivos de las casas comerciales que adquieren los laboratorios clínicos?

$\begin{array}{ccc}\text { PREGUNTA } & \text { SI } & \text { NO } \\ \text { JEFES DE } & 15 & 0 \\ \text { LABORATORIOS } & & \\ \text { ANALISTAS DE } & 28 & 0 \\ \begin{array}{c}\text { LABORATORIOS } \\ \text { TOTAL } \%\end{array} & 100 \% & 0 \%\end{array}$

Del total de la Población encuestada el $100 \%$ conocía del tema mientras que el $0 \%$ No.

4.- ¿Acudiría usted a un laboratorio que trabaje con los Kits de Reactivos Normalizados aplicando una modificación de los mismos?

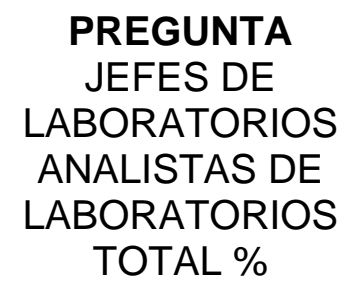

PREGUNTA LABORATORIOS

ANALISTAS DE

TOTAL \%
SI

10

12

$51.16 \%$
NO

5

16

Del total de la Población encuestada el $51.16 \%$ conocía del tema mientras que el $48.84 \%$ No.

5.- ¿Si se hiciera una modificación al método y la validara frente la OAE tendría la confianza de los resultados?

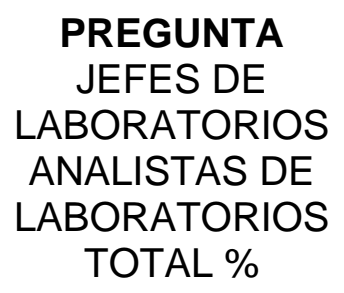

$\begin{array}{cc}\text { SI } & \text { NO } \\ 15 & 0 \\ 28 & 0 \\ & \\ 100 \% & 0 \%\end{array}$

Del total de la Población encuestada el 100\% conocía del tema. 
6.- ¿Sabía usted que los valores de Creatinina en Suero indican el estado de salud de sus riñones?

$\begin{array}{ccc}\text { PREGUNTA } & \text { SI } & \text { NO } \\ \text { JEFES DE } & 15 & 0 \\ \text { LABORATORIOS } & & \\ \begin{array}{c}\text { ANALISTAS DE } \\ \text { LABORATORIOS } \\ \text { TOTAL } \%\end{array} & 26 & 2 \\ & 95.34 \% & 4.66 \%\end{array}$

Del total de la Población encuestada el 95.34 \% conocía del tema mientras que el 4.66 \% No.

7.- ¿Le gustaría que todos los Laboratorios Clínicos del Cantón General Villamil Playas tengan acreditados sus métodos internos?

$\begin{array}{ccc}\text { PREGUNTA } & \text { SI } & \text { NO } \\ \text { JEFES DE } & 15 & 0 \\ \text { LABORATORIOS } & & \\ \text { ANALISTAS DE } & & 0 \\ \begin{array}{c}\text { LABORATORIOS } \\ \text { TOTAL } \%\end{array} & 28 & 0 \%\end{array}$

Del total de la Población encuestada el 100\% conocía del tema mientras que el 0\% No.

8.- ¿Ha considerado en validar sus métodos internos?

$\begin{array}{ccc}\text { PREGUNTA } & \text { SI } & \text { NO } \\ \text { JEFES DE } & 5 & 10 \\ \text { LABORATORIOS } & & \\ \begin{array}{c}\text { ANALISTAS DE } \\ \text { LABORATORIOS }\end{array} & 3 & 25 \\ \text { TOTAL \% } & 18.6 \% & 81.4 \%\end{array}$

Del total de la Población encuestada el 18.6\% conocía del tema mientras que el $81.4 \%$ No. 
9.- ¿Cree usted que son confiables los datos obtenidos a través de una modificación del Método

de Determinación de Creatinina?

$\begin{array}{ccc}\text { PREGUNTA } & \text { SI } & \text { NO } \\ \text { JEFES DE } & 13 & 2 \\ \text { LABORATORIOS } & & \\ \begin{array}{c}\text { ANALISTAS DE } \\ \text { LABORATORIOS } \\ \text { TOTAL } \%\end{array} & 24 & 4 \\ & 86 \% & 14 \%\end{array}$

Del total de la Población encuestada el 86\% conocía del tema mientras que el 14\% No.

10.- ¿Ha trabajado usted con los métodos de Creatinina modificado con los pacientes que acuden a su laboratorio?

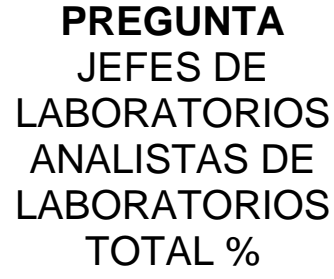

PREGUNTA

LABORATORIOS

ANALISTAS DE

TOTAL \%
SI

14

22

$65.2 \%$
NO

1

6

$34.8 \%$

Del total de la Población encuestada el 65.2\% conocía del tema mientras que el 34.8\% No. 
Dom. Cien., ISSN: 2477-8818

Vol. 4, núm. 1, enero, 2018, pp. 249-261

Validación retrospectiva de la modificación del método bioquímico para la determinación de creatinina en suero, en un laboratorio clínico

\section{Datos Estadísticos de la comparación de resultados}

\begin{tabular}{|c|c|c|c|c|}
\hline \multirow{2}{*}{$\begin{array}{l}\text { TOMAS DE } \\
\text { MUESTRA } \\
\text { SEMANA }\end{array}$} & \multicolumn{2}{|c|}{ METODO MODIFICADO } & \multicolumn{2}{|c|}{ METODO NORMALIZADO } \\
\hline & $\begin{array}{l}\text { Valor Obtenido } \\
\text { en suero mg/l }\end{array}$ & $(x-\bar{x})^{2}$ & $\begin{array}{l}\text { Valor Obtenido } \\
\text { en suero } \mathrm{mg} / \mathrm{l}\end{array}$ & $(x-\bar{x})^{2}$ \\
\hline 1 & 7.26 & 0.0398 & 7.27 & 0.0809 \\
\hline 2 & 7.36 & 0.0099 & 7.35 & 0.0418 \\
\hline 3 & 7.45 & 0.0001 & 7.50 & 0.0030 \\
\hline 4 & 7.46 & 0.0000 & 7.47 & 0.0071 \\
\hline 5 & 7.58 & 0.0145 & 7.56 & 0.0000 \\
\hline 6 & 7.49 & 0.0009 & 7.70 & 0.0212 \\
\hline 7 & 7.33 & 0.0168 & 7.40 & 0.0239 \\
\hline 8 & 7.36 & 0.0099 & 7.20 & 0.1257 \\
\hline 9 & 7.50 & 0.0016 & 7.66 & 0.0111 \\
\hline 10 & 7.53 & 0.0050 & 7.50 & 0.0030 \\
\hline 11 & 7.38 & 0.0063 & 7.40 & 0.0239 \\
\hline 12 & 7.55 & 0.0082 & 7.80 & 0.0603 \\
\hline 13 & 7.36 & 0.0099 & 7.40 & 0.0239 \\
\hline 14 & 7.38 & 0.0063 & 7.44 & 0.0131 \\
\hline 15 & 7.60 & 0.0197 & 7.88 & 0.1060 \\
\hline 16 & 7.39 & 0.0048 & 7.46 & 0.0089 \\
\hline 17 & 7.00 & 0.2111 & 7.85 & 0.0873 \\
\hline 18 & 7.50 & 0.0016 & 7.80 & 0.0603 \\
\hline 19 & 7.86 & 0.1604 & 7.90 & 0.1194 \\
\hline 20 & 7.85 & 0.1525 & 7.55 & 0.0000 \\
\hline
\end{tabular}

\section{Datos Estadísticos del Performance en Reproducibilidad}

\begin{tabular}{|c|c|c|}
\hline NIVEL & D.S. & C.V. \\
\hline $7.3 \mathrm{mg} / \mathrm{l}$ & $\pm 0.18 \mathrm{mg} / \mathrm{l}$ & $2.4 \%$ \\
\hline
\end{tabular}

\section{Performance Método Normalizado VS. Método Modificado}

\begin{tabular}{|l|c|c|}
\hline \multicolumn{1}{|c|}{ Cálculos } & Método Normalizado & Método Modificado \\
\hline $\bar{X}=$ Media & $7.46 \mathrm{mg} /$ & $7.55 \mathrm{mg} / 1$ \\
\hline $\begin{array}{l}\text { S = Desviación } \\
\text { Estándar }\end{array}$ & $\pm 0.19 \mathrm{mg} / \mathrm{l}$ & $\pm 0.21 \mathrm{mg} / \mathrm{l}$ \\
\hline $\begin{array}{l}\text { CV = Coeficiente de } \\
\text { Variación }\end{array}$ & $2.54 \%$ & $2.75 \%$ \\
\hline
\end{tabular}




\section{Comprobación de la hipótesis}

Una vez interpretados los resultados damos por sentado que validar la variación de la técnica de determinación en creatinina en suero nos ayudó a demostrar que los resultados fueron precisos, seguros y confiables. Que servirá de base para la futura acreditación del Laboratorio.

Tanto así que se logró realizar un mayor número de exámenes y se disminuyó el costo de estos, cosa que beneficio mucho a los pobladores de este sector, debido a las limitaciones económicas y la falta de empleo en estas localidades.

\section{Conclusiones.}

Una vez realizado la sistematización de la experiencia se ha llegado a las siguientes conclusiones.

Los resultados obtenidos con el Método Modificado no varían mucho con el de la casa comercial, ya al utilizar la misma muestra con el resultado conocido para ambas corridas, observamos que las dos se encontraban dentro de las especificaciones. Por consiguiente el Método Modificado fue preciso.

De acuerdo a la técnica utilizada en la recolección de información se pudo establecer que el 65\% de los Laboratorios Clínicos del Cantón General Villamil Playas trabajan con el método modificado de la Determinación de Creatinina en Suero. Que el 86\% aseguran que la modificación de la técnica es segura, confiable y precisa.

El Laboratorio Clínico donde se realizó esta investigación ha iniciado la socialización de esta propuesta juntos con los otros laboratorios del Cantón General Villamil Playas para 
entablar una conversación con los representantes de los Reactivos de Wiener Laboratorios S.A. mostrándoles estadísticamente que la Micro técnica empleada de su Reactivo es tan precisa como el Método que viene establecido en los Insertos de los Kits de Reactivos.

Que la población considera que deben acreditarse todos los laboratorios pues ello brindara una mayor confianza en los resultados que reciben y seguramente volverán en caso de la situación lo requiera.

\section{Bibliografía.}

Flores, J., Alvo, M., \& Borja, H. (2009). Enfermedad renal crónica: Clasificación, identificación, manejo y complicaciones. Revista médica de Chile, 137(1), 137-177.

López, F., Blanes, M., \& Ríos, M. (2006). Valoración de Urea, Creatinina y Electrolitos pre y post hemodiálisis en pacientes renales del Hospital Nacional de Itauguá. UNA, 12(1), 26-32.

Myers, G., Miller, W., Coresh, J., Fleming, J., \& Greenberg, N. (2006). Recommendations for improving serum creatinine measurement: A report from the Laboratory Working Group of the National Kidney Disease Education Program. Clin Chem, 1, 5-18.

Pennacchiotti, G., Benozzi, S., \& Ruiz, G. (2012). Impacto de la medición de creatinina en la estimación de la velocidad de filtración glomerular. Acta bioquímica clínica latinoamericana, 46(2), 205-211.

Perazzi, B., \& Angerosa, M. (2011). Creatinina en sangre: calidad analítica e influencia en la estimación del Índice de Filtrado Glomerular. Acta bioquímica clínica latinoamericana, 2, 265-272. 